(C) Facultad de Ciencias Biológicas UNMSM

\title{
Efecto de cuatro ecotipos de Lepidium peruvianum Chacón sobre la producción de óxido nítrico in vitro
}

\author{
Effect of four ecotypes of Lepidium peruvianum Chacón on the \\ production of nitric oxide in vitro
}

(1) Universidad Nacional Mayor de San Marcos, Facultad de Ciencias Biológicas, Instituto de Investigaciones de Ciencias Biológicas Antonio Raimondi. e-mail Libertad Alzamora: lalzamorag@unmsm.edu.pe

(2)Universidad Nacional Mayor de San Marcos, Facultad de Medicina Humana, Instituto de Medicina Tropical Daniel Alcides Carrión.

\section{Libertad Alzamora', Evelyn Alvarez¹, Dina Torres ${ }^{1}$, Hilda Solís², Erasmo Colona", Jenny Quispe ${ }^{1}$ y Magda Chanco ${ }^{1}$}

\section{Resumen}

Los macrófagos desempeñan un rol importante en la respuesta innata y adaptativa, durante su activación producen mediadores citotóxicos como Óxido Nítrico (NO). El objetivo fue evaluar la producción de NO por macrófagos peritoneales de ratón cultivados con extractos metanólicos (EM) de los ecotipos rojo, negro, morado y blanco de Lepidium peruvianum Chacón (también conocida como Lepidium meyenii Walp). Los EM se prepararon empleando maca pulverizada macerada en metanol (1:2) durante 10 días. Los macrófagos peritoneales se obtuvieron de ratones 3 días después de haberles inyectado $1 \mathrm{ml}$ de Caldo Tioglicolato por vía intraperitoneal; se cultivaron por triplicado durante $18 \mathrm{~h}$ a $37^{\circ} \mathrm{C}$ en medio RPMI 1640 suplementado con $10 \%$ de suero de bovino fetal. La dosis de EM fue de $800 \mu \mathrm{g} / \mathrm{ml}$ por ecotipo, se consideraron controles sin EM. La producción de NO se determinó por acumulación de nitrito en el medio y se evidenció con el reactivo de Peter Griess, las concentraciones de nitrito se calcularon en base a la curva estándar elaborada con $\mathrm{NaNO}_{2}$ Las concentraciones producidas de nitrito fueron de 7,45; 6,79; 5,$76 ; 5,61$ y $6,81 \mathrm{mM}$ para los EM de los ecotipos morado, negro, blanco, rojo y control respectivamente. Los cuatro ecotipos indujeron la producción de NO, aunque con el ecotipo morado fue superior $(p>0,05)$.

Palabras Clave: Lepidium peruvianum, Lepidium meyenii, ecotipos de maca, óxido nítrico, macrófagos activados.

\section{Abstract}

The macrophages play an important role in the innate and adaptative response. Upon appropriate activation, macrophages released a variety of cytotoxic mediators like the Nitric Oxide (NO). The objective was to evaluate the production of NO for murine peritoneal macrophages cultivated with methanol extracts (ME) of different ecotypes of Lepidium peruvianum Chacón (at present Lepidium meyenii Walp.) red, black, purple and white. The ME was prepared using maca powdered macerated in methanol (1:2) during 10 days. Peritoneal macrophages were obtained of mice 3 days after having injected them $1 \mathrm{ml}$ of thioglycolate broth; they were cultivated for triplicate during $18 \mathrm{hrs}$ at $37{ }^{\circ} \mathrm{C}$ in RPMl 1640 medium supplemented with $10 \%$ fetal calf serum. The dose of ME was of $800 \mu \mathrm{g} / \mathrm{ml}$ for ecotype, were considered controls without ME. The production of NO was assayed by nitrite accumulation in the supernatant culture and it was evidenced with Peter Griess's reagent, the nitrite concentrations were calculated based on the standard curve elaborated with $\mathrm{NaNO}_{2}$. The produced concentrations of nitrite were of 7,$45 ; 6,79 ; 5,76 ; 5,61$ and $6,81 \mathrm{mM}$ for the ME purple, black, white, red ecotypes and control respectively, although with the purple ecotype it was superior $(p>0,05)$.

Keywords: Lepidium peruvianum, Lepidium meyenii, maca ecotypes, nitric oxide, activated macrophages.

\section{Introducción}

En plantas medicinales han sido encontradas varias clases de metabolitos con propiedades antimutagénicas, antioxidantes, anticancerígenas e inmunomoduladoras, substancias importantes en el tratamiento de varias enfermedades infecciosas, desórdenes inmunológicos y cáncer (Bianchi, 2003; Punture et al., 2004). Lepidium peruvianum Chacón (también conocida como Lepidium meyenii Walp.) «Maca», es una Brassicacea nativa de los Andes Peruanos, que se cultiva principalmente en las regiones Suni y Puna

Nota del Editor: El nombre Lepidium peruvianum Chacón debe ser considerado sinónimo de Lepidium meyenii Walp. (ver Germplasm Resources Information Network, United States Department of Agriculture, Agricultural Research Service, en http:/ /www.ars-grin.gov/cgi-bin/npgs/html/taxon.pl?21767, acceso 08/02/07) preferencias del autor obligan a mantener L. peruvianum Chacón en el texto. de los departamentos de Junín y Pasco, entre los 3700 y 4500 m. Su raíz constituye la porción comestible de la planta, y la medicina tradicional le atribuye propiedades afrodisíaca, reguladora, inmunoestimulante, energizante y antiartrítica (Obregón, 1998; Bianchi, 2003). Estudios sobre sus metabolitos secundarios señalan la presencia de alcaloides, esteroides, glucosinolatos, isotiocianatos y macamidas, sin embargo es poco lo que se conoce sobre los mecanismos de acción de estos metabolitos y sobre todo su asociación con los efectos atribuidos a la planta (Muhammad et al., 2002; Valentová y Ulrichová, 2003).

Se han descrito diferentes ecotipos de Maca considerando el color de su raíz, siendo el ecotipo amarillo el más frecuente $(47,8 \%)$ y comercializado (Obregón, 1998). Estudios comparativos de los ecotipos amarillo, rojo y negro señalan diferencias nutricionales entre ellos (Yllesca, 1994). Además, recientemente se reportaron 
diferencias farmacológicas sobre la espermatogénesis y el tamaño de la próstata de tres ecotipos de maca. El tamaño de la próstata se redujo significativamente en ratas tratadas con maca roja luego de dos inoculaciones de enantato de testosterona (Gonzales G. et al., 2006; Gonzales G. et al., 2005).

Los macrófagos son células importantes en el desarrollo de la inmunidad innata y adaptativa. Cuando son activados ya sea por efecto de citoquinas pro-inflamatorias o infecciones con microorganismos, liberan Óxido Nítrico (NO) y otros intermediarios reactivos del oxígeno, responsables de su actividad citotóxica y citostática contra microorganismos infecciosos y células tumorales (Tamez et al., 2001; Ignacio et al., 2001).

Sandoval et al., (2002) reportaron el efecto antioxidante de un extracto acuoso de maca, evidenciado por su capacidad citoprotectora contra la apoptosis inducida. Esto indicaría que la actividad citotóxica es selectiva y está dirigida contra células tumorales. También hemos observado actividad antitumoral e inmunomoduladora de los extractos acuoso, metanólico y alcaloidal de la maca-ecotipo amarillo, atribuida a la estimulación de macrófagos (incremento de fagocitosis y producción de NO) y otras células del sistema inmune (Alzamora et al., 2007a y 2007b).

El presente estudio evaluó in vitro el efecto del extracto metanólico de los ecotipos rojo, blanco, morado y negro de Lepidium peruvianum Chacón (Lepidium meyenii Walp.) sobre la producción de óxido nítrico como evidencia de la activación de los macrófagos.

\section{Material y métodos}

Se emplearon raíces de Lepidium perwianum procedentes del Valle de Pampas, Provincia de Tayacaja, Departamento de Huancavelica, las que fueron clasificadas por ecotipos según su color y teniendo en cuenta su forma y estado de conservación. Se seleccionaron cuatro ecotipos de maca: rojo, blanco, morado y negro.

Las raíces se pulverizaron y maceraron en metanol QP durante 10 días (1:2 P/V). El macerado se filtró y se concentró en una estufa de aire circulante a $40^{\circ} \mathrm{C}$ hasta total evaporación del solvente.

Se empleó la dosis de $800 \mu \mathrm{g} / \mathrm{ml}$. Para su preparación se pesó el extracto obtenido y se diluyó en medio RPMI-1640, el preparado se esterilizó empleando filtro milipore $0,20 \mu$. El filtrado de cada ecotipo se almacenó a $10^{\circ} \mathrm{C}$ hasta su uso.

Se trabajó con ratones machos Swiss de 8 semanas de edad, obtenidos del bioterio de la Facultad de Ciencias Biológicas, UNMSM. Los ratones se criaron bajo condiciones fisiológicas.

Los macrófagos peritoneales se obtuvieron, por lavado de la cavidad peritoneal, de ratones previamente inoculados con $1 \mathrm{ml}$ de Caldo Tioglicolato (CT) estéril i.p. tres días antes de la evaluación (dosis única). La cavidad peritoneal se lavó con $3 \mathrm{ml}$ de RPMI1640, el volumen recolectado se depositó en tubos, se centrifugaron a $1000 \mathrm{rpm}$ por 7 minutos, los botones se resuspendieron en RPMI-1640 suplementado con suero bovino fetal $10 \%$, penicilina $(100 \mathrm{UI} / \mathrm{ml})$ y estreptomicina $(100 \mu \mathrm{g} / \mathrm{ml})$, conteniendo el extracto metanólico (EM) a la dosis de $800 \mu \mathrm{g} / \mathrm{ml}$, se consideró como control los cultivos de macrófagos sin EM. Los cultivos (por triplicado) se incubaron durante $18 \mathrm{~h} \mathrm{a} 37^{\circ} \mathrm{C}$.

A las $18 \mathrm{~h}$ de cultivo se determinaron los niveles de nitrito para cada ecotipo por medio de la reacción de Griess. Para ello, se mezclaron $100 \mu \mathrm{l}$ del sobrenadante de cada cultivo con $100 \mu \mathrm{l}$ del Reactivo de Griess ( $50 \mathrm{ml}$ de Solución A + $50 \mathrm{ml}$ de Solución B). Se midió la absorbancia de la mezcla a $540 \mathrm{~nm}$. La concentración de nitrito se determinó usando como estándar una curva de nitrito de sodio.

Los resultados se expresaron como la media \pm desviación estándar de las determinaciones por triplicado. Los datos fueron analizados utilizando la prueba t de Student.

\section{Resultados}

La inoculación de Caldo Tioglicolato indujo un incremento en la población de macrófagos peritoneales, esta técnica es muy utilizada para la colección de mayor cantidad de estas células (McCarron et al., 1984), el medio actúa como un estímulo inflamatorio no específico. Las concentraciones producidas de nitrito fueron de 7,45; 6,79; 5,76; 5,61 y 6,81 mM para los EM de los ecotipos morado, negro, blanco, rojo y control respectivamente.

El EM de cada ecotipo indujo la producción de óxido nítrico, aunque las diferencias entre los tratamientos no mostraron diferencias significativas $(\mathrm{p}>0,05)$. Aunque al compararse con el control (Fig. 1) se observó un ligero incremento con el EM del ecotipo morado y disminución con el EM del ecotipo rojo. También se observó producción de óxido nítrico en el control (sin EM) debido al uso de caldo tioglicolato como atrayente que permite concentrar una mayor cantidad de macrófagos (agente inflamatorio inespecífico).

\section{Discusión}

Actualmente, se estima que alrededor del $80 \%$ de la población mundial recurre a la medicina tradicional para la atención primaria de la salud. Siendo la maca una planta con probadas propiedades nutricionales este trabajo contribuye con incrementar el valor agregado del recurso ya que se demuestra que el ecotipo morado ha logrado inducir mayor producción de óxido nítrico.

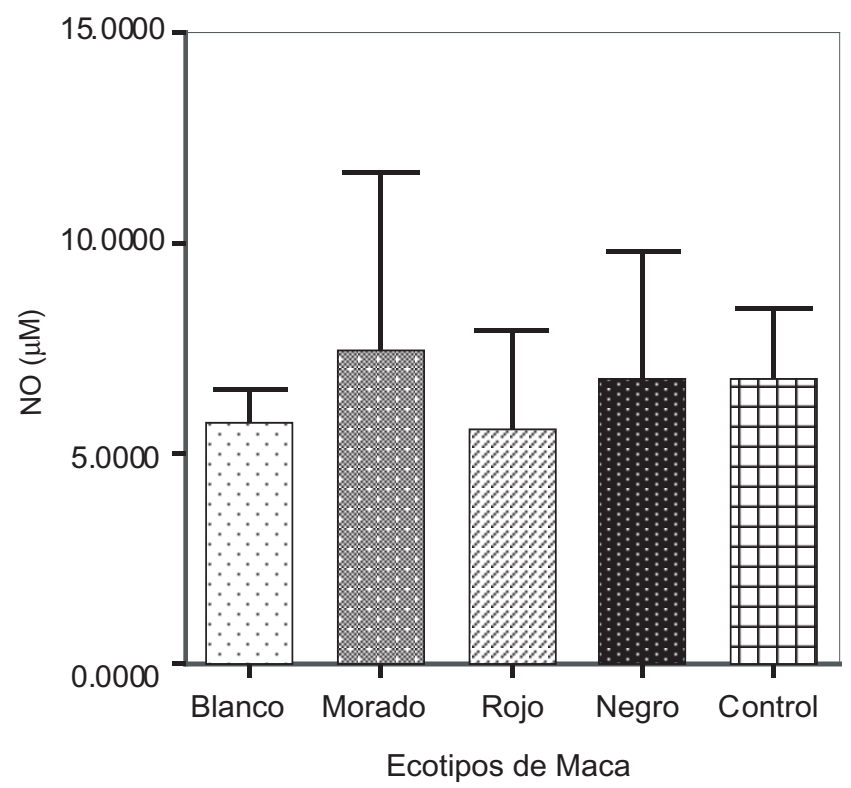

Figura 1. Efecto del Extracto Metanólico (EM) de los ecotipos rojo, blanco, morado y negro de Lepidium peruvianum, Maca, sobre la producción de Óxido Nítrico por macrófagos peritoneales de ratón tratados con $800 \mu \mathrm{g} / \mathrm{ml}$ de EM. Los cultivos se mantuvieron durante 18 horas, los niveles de óxido nítrico se determinaron por acumulación de nitritos en los sobrenadantes. Se observa mayor incremento en la producción de NO inducida por el EM del ecotipo morado y menor en el caso del ecotipo rojo ( $p>0,05)$. Los datos representan la media \pm la desviación estándar de los niveles de nitrito. 
Del mismo modo, se debe resaltar la menor producción de NO en los cultivos con el EM del ecotipo rojo. En el caso del ecotipo morado, Alzamora et al. (2007a) determinaron que este ecotipo también estimulaba una mayor producción de IFN- $\gamma$ por cultivos de linfocitos humanos; por lo tanto el efecto sería de amplio espectro. Respecto al ecotipo rojo, el efecto antiinflamatorio sobre la próstata en ratas fue observado por Gonzales et al., (2005), y podría estar relacionado con la menor producción de NO encontrada en el presente estudio.

En conclusión los extractos metanólicos de los ecotipos rojo, blanco, morado y negro a la dosis de $800 \mu \mathrm{g} / \mathrm{ml}$ inducen la producción de óxido nítrico por los cultivos de macrófagos de manera similar al control.

\section{Agradecimientos}

Los autores agradecen el financiamiento otorgado por el Consejo Superior de Investigaciones-UNMSM (Proyecto $\mathrm{N}^{\circ}$ 051001061).

\section{Literatura citada}

Alzamora L., P. Galván, E. Alvarez, D. Torres, E. Colona, M. Aliaga \& A. Marcelo. Producción de IFN-g en cultivos de linfocitos humanos por efecto de los extractos metanólicos de cuatro ecotipos de Lepidium peruvianum, Chacón (Brassicaceae). 2007a. Rev. peru. biol. Número especial 13(3): 000- 000

Alzamora L., H. Solís, M. Rojas, M. Calderón, N. Fajardo, J. Quispe, E. Alvarez, E. Colona \& D. Torres. Actividad leishmanicida de los extractos metanólicos de cuatro ecotipos de Lepidium peruvianum, Chacón (Brassicaceae). 2007b. Rev. peru. biol. Número especial 13(3): 000- 000

Bianchi, Antonio. 2003. Maca Lepidium meyenii. Boletín Latinoamericano y del Caribe de plantas medicinales y aromáticas 2(3):26-44.

Gonzales, C., J. Rubio, M. Gasco, J. Nieto, S. Yucra y G. Gonzales. 2006. Effect of short-term and long-term treatments with three ecotypes of Lepidium meyenii (MACA) on spermatogenesis in rats. Journal of Ethnopharmacology 103:448-454
Gonzales, G., S. Miranda, J. Nieto, G. Fernández, S. Yucra, J. Rubio, P. Yi y M. Gasco. 2005. Red maca (Lepidium meyenii) reduced prostate size in rats. Reproductive Biology and Endocrinology 3(1):5.

Ignacio, S., J. Ferreira, M. Almeida y C. Kubelka. 2001. Nitric oxide production by murine peritoneal macrophages in vitro and in vivo treated with Phyllanthus tenellus extracts. Journal of Ethnopharmacology 74:181-187.

McCarron, R., D. Goroff, J. Luhr, M. Murphy y H. Herscowitz. 1984. Methods for the collection of peritoneal and alveolar macrophages. Methods in Enzimology 108:274-284.

Muhammad, I., J. Zhao, D. Chuck y I. Khan. 2002. Constituents of Lepidium meyenii (maca). Phytochemistry 59:105-110.

Obregón Vilches, Lida. 1998. «Maca» Planta medicinal y nutritiva del Perú. Instituto de Fitoterapia Americano. 1era edición. Lima-Perú.

Punture, K., C. Wild y V. Vinitketkumneun. 2004. Thai medicinal plants modulate nitric oxide and tumor necrosis factor-a in J774.2 mouse macrophages. J. of Ethnopharmacology 95:183-189.

Tamez, R., C. Rodríguez, P. Tamez, R. Weber, R. Gómez y C. Calderón. 2001. Activación de macrófagos y linfocitos in vitro por extractos metanólicos de hojas de Plantago major. Ciencia UANL. Vol. IV, $\mathrm{N}^{\circ} 3$.

Sandoval, M., N. Okuhama, F. Angeles, V. Melchor, L. Candezo, J. Lao y M. Miller. 2002. Antioxidant activity of the cruciferous vegetable Maca (Lepidium meyenii). Food Chemistry 79: 207-213.

Yllesca Gutierrez, María Gregoria. 1994. Estudio Químico y Fitoquímico comparativo de 3 ecotipos de Lepidium meyenii Walp. «Maca» procedentes de Carhuamayo (Junín). Cátedra de Bromatología; Facultad de Farmacia y Bioquímica, Universidad Nacional Mayor de San Marcos. Lima. 103 pág.

Valentová, K. y J. Ulrichová. 2003. Smallanthus sonchifolius and Lepidium meyenii- Prospective andean crops for the prevention of chronic diseases. Biomed. Paper 147(2):119130. 


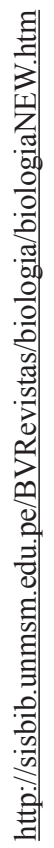

